\title{
Automated ClaSSifiCATiOn OF Similar TWO-DimenSIONAL OBJECTS WITH NEURAL NETWORK APPLICATION
}

\author{
Irina Topalova \& Vyara Tatarska
}
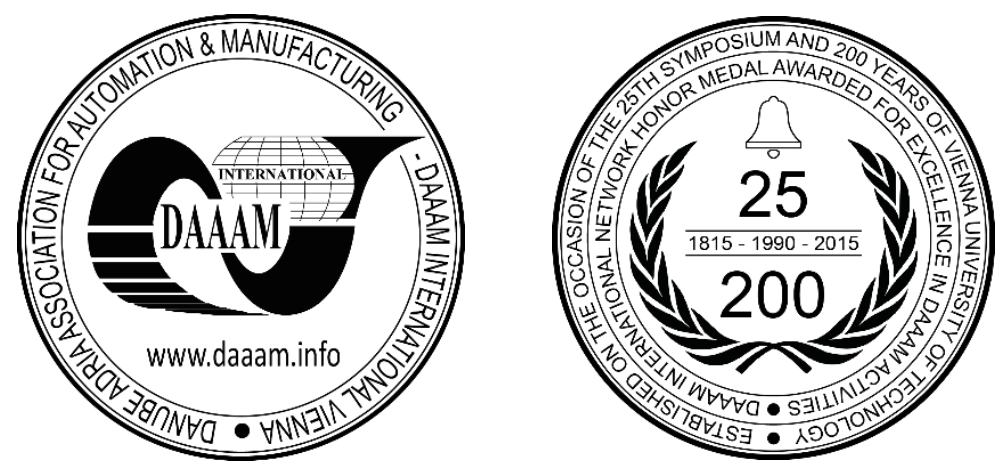

This Publication has to be referred as: Topalova, I[rina] \& Tatarska, V[yara] (2018). Automated Classification of Similar Two-dimensional Objects with Neural Network Application, Proceedings of the 29th DAAAM International Symposium, pp.0225-0231, B. Katalinic (Ed.), Published by DAAAM International, ISBN 978-3-902734-20-4, ISSN 1726-9679, Vienna, Austria

DOI: $10.2507 / 29$ th.daaam.proceedings.032

\begin{abstract}
Very often in automated industrial systems it is necessary to identify and classify very similar two-dimensional objects. This is especially important when determining the quality of the objects and finding defects on their surface and external contour. In these cases, the parametric descriptions of objects strongly overlap and it is necessary to find an effective method for their de-correlation since they are inputs of the recognition system. In this way, the recognition system will be assisted in the decision-making process during the pre-processing/preparation phase of the input data. In this study a method is proposed for determining the parametric descriptions of similar two-dimensional objects and analysis of the similarity between them, with subsequent reduction and de-correlation of these data. Thus, the data prepared are submitted for training at the input of an adaptive recognition system, consisting of a multilayer neural network. Experiments with different topologies and neural network parameters were performed to optimize the accuracy of the recognition in the testing phase. The results obtained show significantly higher recognition accuracy when using the developed method for preliminary analysis, reduction and de-correlation of the input data. The results obtained are discussed and the further intentions for the continuation of the study are outlined.
\end{abstract}

Keywords: recognition; 2D objects; correlation; neural network

\section{Introduction}

The task of identifying, recognizing and classifying similar two-dimensional objects is of particular importance to automated industrial systems, especially when determining the quality of their surfaces or defects in the contour of the object. In this case, the parametric descriptions of the objects that are fed to the input of the recognition system overlap. To "facilitate" the recognition system, the input data need to be analyzed and if possible, to be de-correlated in the preprocessing stage. In this research a method is proposed for determining the parametric descriptions of similar twodimensional objects and analysis of the similarity between them, with subsequent reduction and de-correlation of these data. Thus, the data prepared are submitted for training at the input of an adaptive recognition system, consisting of a multilayer neural network. Experiments with different topologies and neural network parameters were performed to optimize the accuracy of the recognition in the testing phase. The results obtained show significantly higher recognition accuracy when using the developed method for preliminary analysis, reduction and de-correlation of the input data. The results obtained are discussed and the further research is outlined. 
The proposed paper is organized as follows. Section 2. describes related to the research works. Section 3. describes the proposed pre-processing method and the investigated neural network topology. Section 4. gives the experimental results. The conclusion closes the article.

\section{Related works}

The appropriate preprocessing of data input for the recognition system is essential for the required recognition accuracy. Many different methods for retrieving parametric descriptions of two-dimensional objects are developed. But when these descriptions represent similar objects, they will be highly correlated. In this case, the recognition system will be "embarrassed" to distinguish their similarity. For example, in [1] the authors represent texture-based image classification using the gray-level co-occurrence matrices (GLCM) and self-organizing map (SOM) methods applied for very similar textures. They show the superiority of GLCM+SOM over the single and fused Support-Vector Machine (SVM), over the Bayes classifiers using Bayes distance and Mahalanobis distance. The authors obtain $97.8 \%$ accuracy but the use of GLCM needs high computations and even faster version of a Co-occurrence matrix as given in [2], needs computations multiple times over the whole image for each of the three colours. The authors of [2] constitute a neural network (NN) input feature vector of mean, energy, entropy, contrast and homogeneity, for each of the three colour channels. In this case the authors claim high-speed processing but the obtained recognition accuracy is $80-92.7 \%$. The calculation of Wavelets over the parametric descriptions, using hierarchical NN structure, feeding different NNs [3,4] with different input feature vectors, would be more complicated, because of time-consuming operations, particularly for real-time applications in hardware platforms. The authors of [5] rather does a comparative analysis of existing methods for pre-processing of images in stages "segmentation", "selection and defining of object description parameters" and "classification" without a subsequent prescription for a suitable combination of methods for recognizing very similar objects. By choosing to combine multiple images from different cameras into a single high-resolution stream, the authors of [6] have succeeded to integrate a distributed system across the entire production line, using a single an application to detect, classify and count all products in different manufacturing stages. They benefit from a fastest detection platform which allows real time classification at a small price, applying convolutional networks. This method is productive when a variety of objects are recognized when changing production, but does not discuss the case of an effective differentiation when these objects are very similar. Thus, the important source of optimizing the recognition method, when it comes to finding small differences between objects, lies in the analysis of the degree of correlation between their parametric descriptions, in the subsequent simplification of the input feature vectors and last but not least in finding more efficient training method along with reducing the NN nodes.

\section{The proposed method}

The proposed method for recognizing two-dimensional objects with small defects in the contour includes determining the radial profile (RP) of the objects, which is chosen to be their parametric description. In the next step, the description/signal is subdivided into ranges to calculate the correlation for each range individually, between the ideal and each of the other defective objects. Then, in order to reduce the signal size, only the range in which the correlation is lowest is taken into account. Next, suitable mathematical signal conversion is sought for additional de-coloration to reduce further the similarity between the radial profiles. Thus, the de-correlated data form an input vector for training a multilayer neural network (MLP-NN).

\subsection{Calculation of the radial profiles}

To make the experiment more accurate and productive, 5 objects with different defect sizes were created. Each defect starts at $5 \mathrm{~mm}$ of the upper left side and increases with a step of $2.5 \mathrm{~mm}$ for each next object.

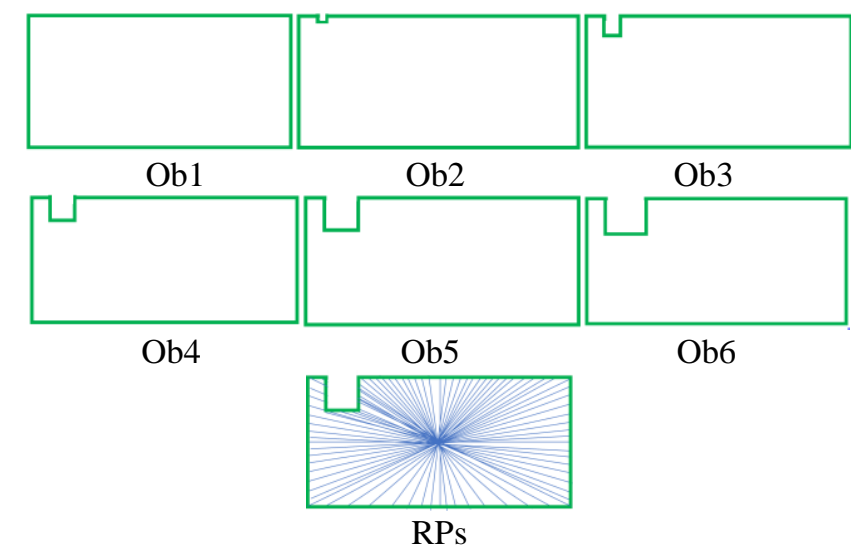

Fig. 1. Experimental objects with following defects: Ob1-ideal, Ob2-2.5x2.5mm, Ob3-5x5mm, Ob4-7.5x7.5mm, Ob510x10mm, Ob6-12.5x12.5mm 
The first object, which from now on is called Ob1, has no any defect. It serves as the basis for comparison with other objects. The size of the ideal object is $\mathrm{a}=80, \mathrm{~b}=40 \mathrm{~mm}$. The millimeters are the more preferred unit of measurement than centimeters, as they will provide more accurate data to the experiments. Figure 1 shows the experimental objects with following defect sizes: Ob1-ideal, Ob2-2.5x2.5mm Ob3-5x5mm, Ob4-7.5x7.5mm, Ob5-10x10mm, Ob6$12.5 \times 12.5 \mathrm{~mm}$. It means that the smallest defect represents $0.19 \%$ of the total site area.

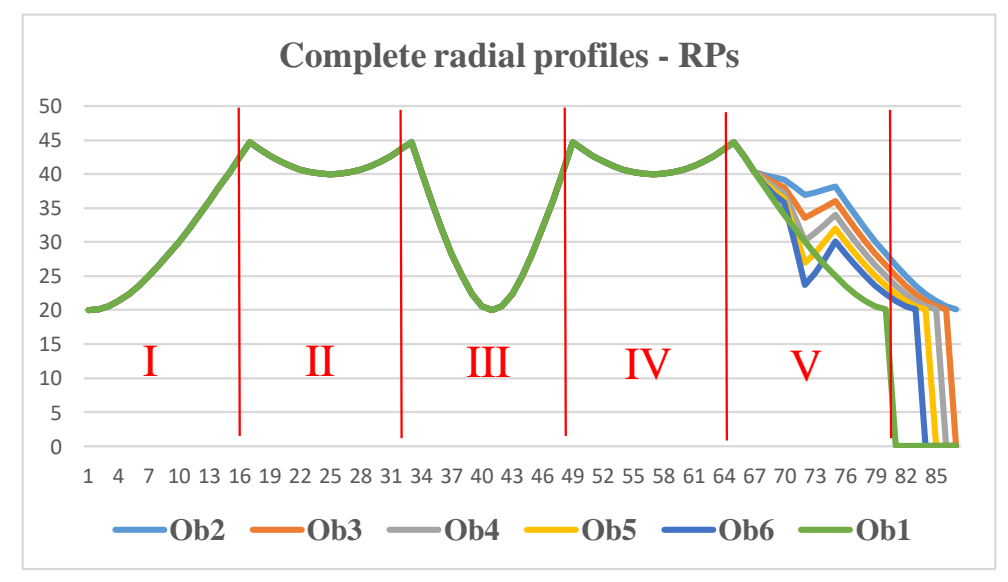

Fig. 2. Calculated radial profiles for the experimental objects

The radial profiles (RPs) are formed using radius vectors. They are taken by finding the center of gravity (the intersection of the diagonals) of each object and constructing them at different points of the contour. Each object is divided into 4 quadrants and the defect is always in upper left quadrant, to simulate a defect generated for technological reasons. Furthermore, for more accurate results, the vectors are plotted at a denser distance and at the same angle from each other. The obtained RPs are represented in Figure 2.

\subsection{Correlation analysis}

The next goal is to reduce the size of PPs without losing the signal informativeness. It is logical to try to find those areas in which PPs of individual sites differ most strongly, i.e. they are weak correlated. For our experiment, we divide the signal into five identical regions, and for each the correlation between the RP of Ob1 and RP of each of the defective objects was calculated. The correlation has been calculated using the Pearson correlation coefficient [7], because it considers the statistical features of the signal:

$C C_{1 k}=\frac{\sum_{i=1}^{n}\left(R P_{1 i}-\overline{R P_{1}}\right) \cdot\left(R P_{k i}-\overline{R P_{k}}\right)}{\sqrt{\sum_{1}^{n}\left(R P_{1 i}-\overline{R P_{1}}\right)^{2} \cdot\left(R P_{k i}-\overline{R P_{k}}\right)^{2}}}$, where

$R P_{l i}$ is the $i$-th component of $\mathrm{PR}$ for $\mathrm{Ob} 1, \overline{R P_{1}}$ is the mean value of its components; $R P_{k i}$ is the $i$-th component of PR for $\mathrm{Ob} k, \overline{R P_{k}}$ is the mean value of its components. The obtained results for correlation $C C_{l k}$ as can be expected was very high, between 0.99 and 0.98 for regions I to IV in Figure 1. For region V, the signal differences reflect in lower correlation between the obtained RPs. They are between 0.847 and 0.842 therefore, this portion of the signal would be more suitable for input to the recognition system. The signals in the so-separated region $\mathrm{V}$ show a lower correlation between individual objects, but it is still too high to expect good results from the recognition system.

In this step we are intended to find a mathematical function, applied over RPs, aiming to apply a simple calculation that does not increase the computational resources and at the same time substantially to reduce the correlation between the RPs (only for region V). Such a transformation was used by the author in [8], but for any different statistical distribution of the input data, appropriate mathematical transformations should be sought.

After testing different mathematical transformations on RPs, the best de-correlation was obtained for $\operatorname{Exp}\left(100 / R P_{i}\right)$. Table I shows the obtained correlation coefficient between objects A-B for both signals: only $R P$ and $E X P(100 / R P)$. The resulting substantial de-coloration between RPs is in the range of 0.554 to 0.369 . This result justifies the choice of $\operatorname{EXP}(100 / R P)$ signals to be used as inputs to the recognition system.

\begin{tabular}{|c|c|c|c|c|c|}
\hline Objects A-B & $\mathbf{1 - 2}$ & $\mathbf{1 - 3}$ & $\mathbf{1 - 4}$ & $\mathbf{1 - 5}$ & $\mathbf{1 - 6}$ \\
\hline RP & 0.847189 & 0.84624 & 0.845094 & 0.843687 & 0.842045 \\
\hline EXP(100/RP) & 0.553917 & 0.524854 & 0.485856 & 0.43438 & 0.369066 \\
\hline
\end{tabular}

Table 1. Correlation coefficient between objects A-B for both signals: only $R P$ and $E X P(100 / R P)$ 


\subsection{MLP train method}

After the input data size has been reduced and a de-correlation has been selected, an appropriate recognition method/system should be applied. The advantages of MLP-NN are well known in terms of recognition efficiency sought, especially when it comes to distinguishing similar/highly correlated input data. This is due to their ability to fine-tune boundaries between classes, depending on the number of layers and the neurons in them [9], [10]. From the defined region $\mathrm{V}$, we select 8 values that form the input vector for MLP-NN training.

The structure of MLP-NN 8-7-6 is selected, with 7 neurons in the hidden layer and 6 neurons corresponding to the number of objects recognized, in the output layer. To compare the results with respect to recognition accuracy, the neural network was initially trained with $R P s$, then with $E X P(100 / R P)$ values, which are shown in Figure 3 and Figure 4 respectively. MLP-NN was trained using the Backpropagation (BGP) algorithm through successive reductions of the specified Mean-Squere-Error (MSE), beginning with MSE=0.1 and fine tuning to MSE=0.002, when supplying the input vectors in a random order.

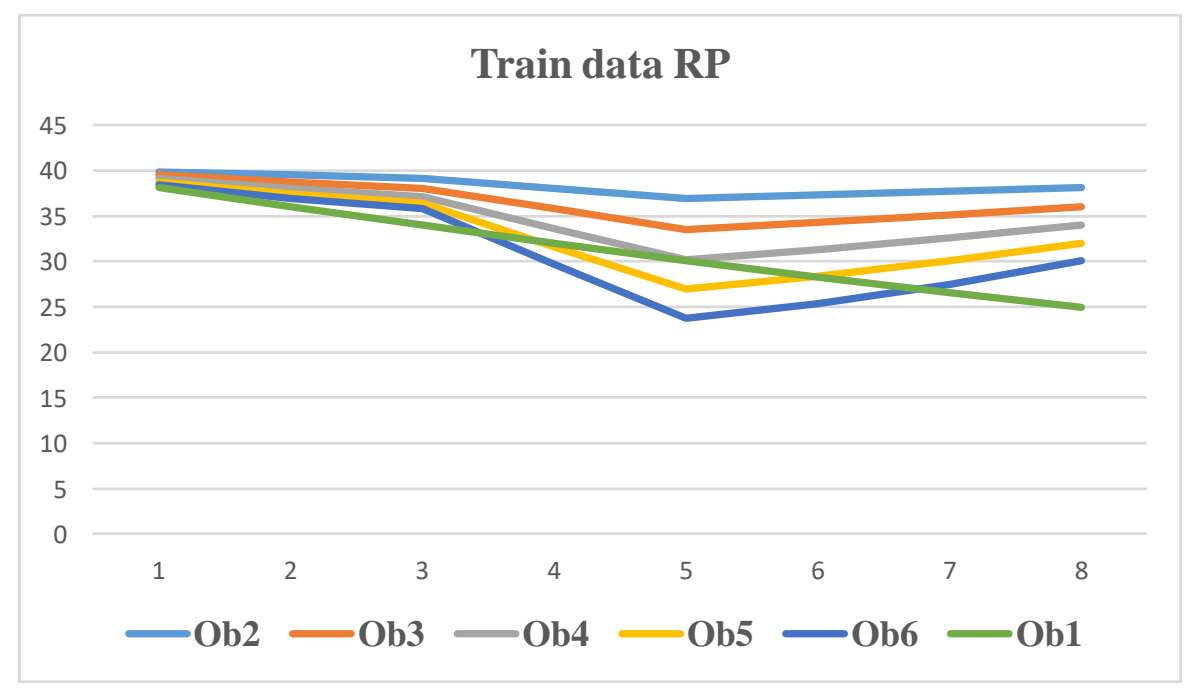

Fig. 3. Training data values with RPs for objects 1 to 6

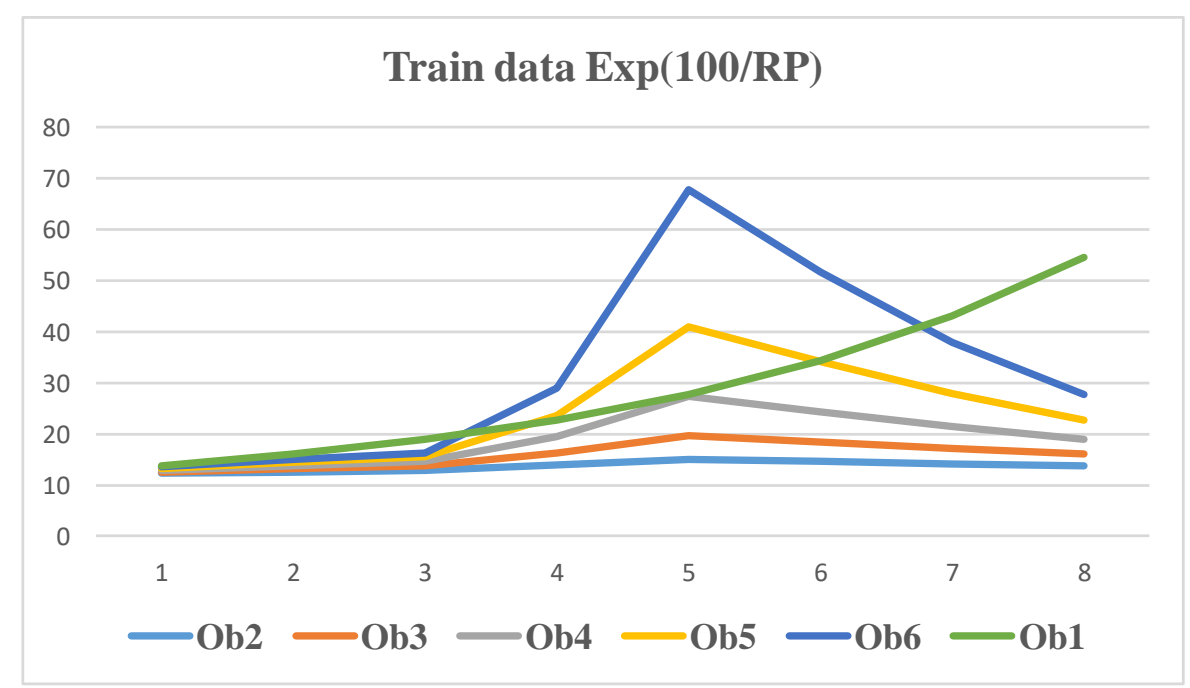

Fig. 4. Training data values with $\operatorname{EXP}(100 / R P)$ for objects 1 to 6

\section{Experimental results for object recognition}

The trained with both types of input vectors MLP-NN, was tested with the relevant type of test representatives who have not participated in the train sampling set. The test specimens were generated by adding positive and negative values to the components of the training data, representing $5 \%$ of the average of the components of the respective training vectors. These test data are represented in Figure 5 and 6 for RPs and $E X P(100 / R P)$ correspondingly. 


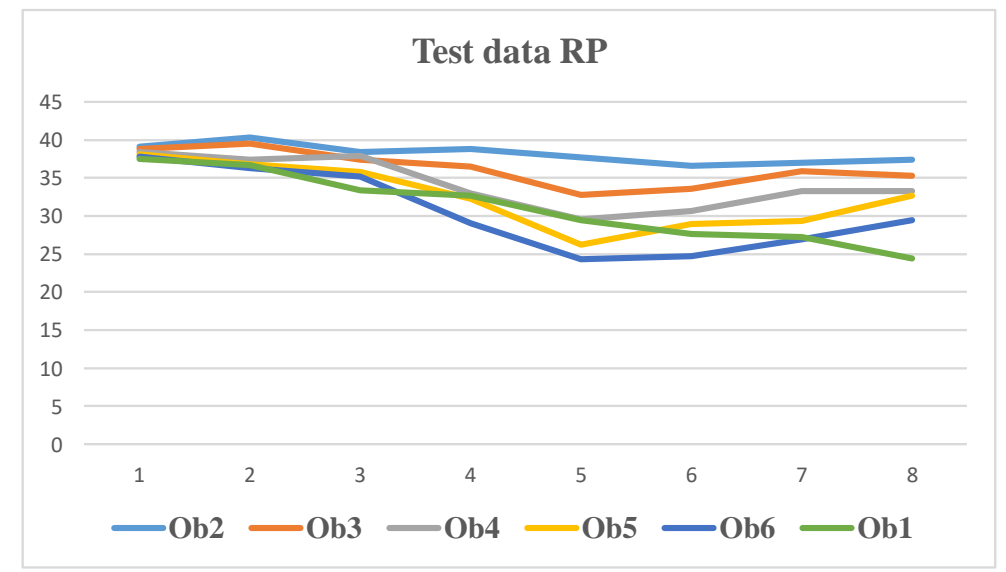

Fig. 5. Test data values with RPs for objects 1 to 6

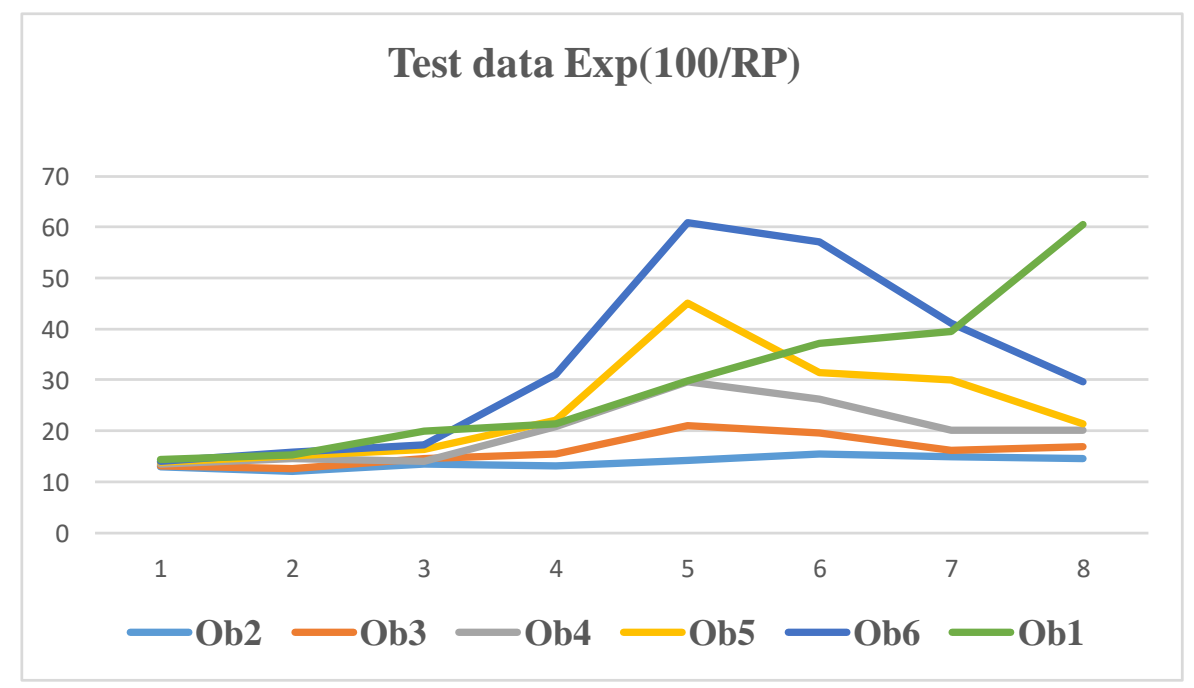

Fig. 6. Test data values with $\operatorname{EXP}(100 / R P)$ for objects 1 to 6

The approximation/recognition accuracy was calculated according to (2)

App_accuracy_neuron $n_{j}=\frac{\sum_{i=1}^{6}\left(N_{i d v}-N_{i r v}\right)^{2}}{6}$,

where $N_{i d v}$ are the desired values and $N_{i r v}$ are the real values components of the MLP output vector. Table 2 represents the results of calculated approximation accuracy for each output neuron value according to (2).

\begin{tabular}{|c|c|c|c|c|c|c|}
\hline & \multicolumn{5}{|c|}{ Aproximation accuracy for neuron $\mathrm{Nj}$ (MLP 8-7-6) } & \multirow[t]{2}{*}{ MSE error } \\
\hline N1 & $\mathrm{N} 2$ & N3 & $\mathrm{N} 4$ & N5 & N6 & \\
\hline 0.09680144 & 1.31862178 & 0.81816939 & 0.60182791 & 0.491996 & 0.22842919 & 0.001 \\
\hline & \multicolumn{5}{|c|}{ Aproximation accuracy for neuron $\mathrm{Nj}$ (MLP 8-6-6) } & \\
\hline N1 & $\mathrm{N} 2$ & N3 & $\mathrm{N} 4$ & N5 & N6 & \\
\hline 0.45507328 & 1.42869299 & 1.3973407 & 1.31900809 & 0.06373369 & 0.01932297 & 0.002 \\
\hline & \multicolumn{5}{|c|}{ Aproximation accuracy for neuron $\mathrm{Nj}$ (MLP 8-7-6) } & \\
\hline N1 & $\mathrm{N} 2$ & N3 & $\mathrm{N} 4$ & N5 & N6 & \\
\hline 0.14493839 & 0.2596984 & 0.78145927 & 1.32303032 & 1.01919107 & 0.12439099 & 0.002 \\
\hline
\end{tabular}

Table 2. Approximation accuracy for neuron $N_{j}$ in the case train and test phase with $E X P(100 / R P)$

In the case of training and testing with RPs, all objects, except Ob5 and Ob3 are recognized correctly. In the case of training and testing with $E X P(100 / R P)$, all objects are recognized correctly. Figure 7 . shows the potential values of the MLP output neurons when applying $R P_{5}$ on the MLP input. The brighter lines show $N_{i d v}$, and the darker ones - $N_{i r v}$. Obviously Ob5 is false recognized. Figure 8. shows the same MLP outputs when applying $\operatorname{EXP}\left(100 / R_{5}\right)$ on the MLP input. In this case Ob5 is true recognized. 


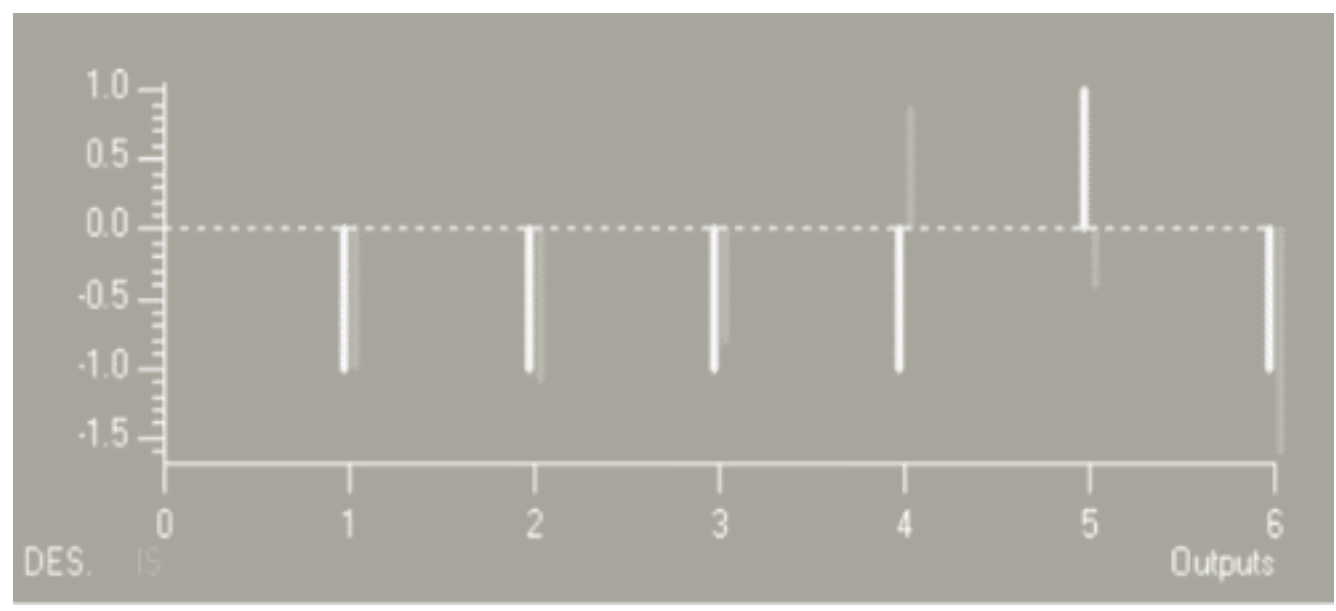

Fig. 7. False recognition of $\mathrm{Ob} 5\left(\mathrm{~N}_{5}\right)$ (in the case train with $\left.R P s\right)$

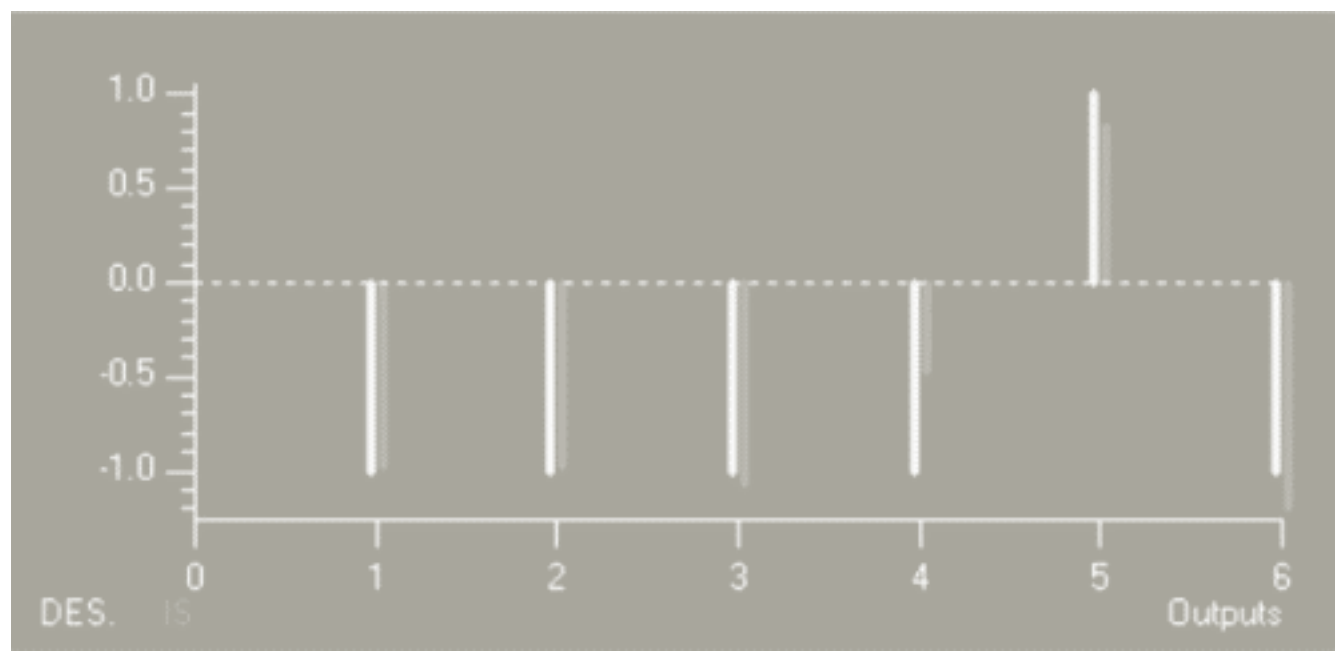

Fig. 8. True recognition of $\mathrm{Ob} 5\left(\mathrm{~N}_{5}\right)$ (in the case train with $\operatorname{EXP}(100 / R P)$ )

\section{Conclusion}

In the represented research a method for determining the parametric descriptions of similar two-dimensional objects and analysis of the similarity between them, with subsequent reduction and de-correlation of these data was proposed. The he data prepared are submitted for training at the input of a MLP-NN. The results obtained when using EXP(100/RP)) as train and test set, show significantly higher recognition accuracy when using the developed method for preliminary analysis, reduction and de-correlation of the input data. For efficient recognition of highly similar objects, it helps to reduce the input data, by selecting only the area for which the parametric descriptions of individual objects are less correlated. On the other hand, this is also due to the preliminary de-correlation of the data in the pre-processing stage. In this case, an appropriate and simple mathematical transformation was found, which does not require large computational resources and would provide the necessary performance needed for real-time applications. For the future continuation of the study, it is envisaged to test the presented method with more samples of real objects with defects also in the internal holes of the objects. In this case, the calculated RPs of the holes will feed a separate NN. The results of the individual NNs will be analyzed appropriately.

\section{References}

[1] Almeida, C.W.D.; de Souza, R.M.C.R. \& Candeias, A.L.B. (2010). Texture Classification Based on a CoOccurrence Matrix and Self-Organizing Map. IEEE International Conference on Systems Man \& Cybernetics, University of Pernambuco, Recife, http://dx.doi.org/10.1109/icsmc.2010.5641934.

[2] Mart'ınez-Cabeza-de-Vaca-Alajar'ın, J.; Tom'as-Balibrea \&Luis-Manuel (1999). Marble Slabs Quality Classifcation System using Texture Recognition and NNs Methodology, ESANN'1999 proceedings - European Symposium on Artificial NNs, Bruges (Belgium), 21-23, D-Facto public., pp. 75-80, ISBN 2-600049-9-X. 
[3] Topalova, I. (2012). Automated Marble Plate Classification System Based on Different NN Input Training Sets and PLC Implementation, IJARAI - International Journal of Advanced Research in Artificial Intelligence, May, pp. 5056, Volume1, Issue2, ISSN: 2165-4069.

[4] Topalova, I. (2015). Recognition of Similar Wooden Surfaces with a Hierarchical NN Structure, SAI/ IJARAI International Journal of Advanced Research in Artificial Intelligence, October, pp. 35-39, Volume 4 Issue10, U.S ISSN: 2165-4069.

[5] Adamek, M.; Neumann, P.,Pospisilik, M., Spanko, A. \& Vasek, V. (2017). Object Recognition in Images, Proceedings of the 28th DAAAM International Symposium, B. Katalinic (Ed.), Published by DAAAM International, pp.1178-1184, ISBN 978-3-902734-11-2, ISSN 1726-9679, Vienna, Austria.

[6] Deac, C. N.; Popa, C. L., Ghinea, M. \& Cotet, C. E. (2017). Machine Vision in Manufacturing Processes and the Digital Twin of Manufacturing Architectures, Proceedings of the 28th DAAAM International Symposium, B. Katalinic (Ed.), Published by DAAAM International, pp.0733-0736, ISBN 978-3-902734-11-2, ISSN 1726-9679, Vienna, Austria.

[7] Rodgers, J. L. \& Nicewander, W. A. (Accessed September 2018). "Thirteen ways to look at the correlation coefficient," The American Statistician, vol. 42, no. 1, pp. 59-66, 1988. [Online]. Available: https://doi.org/10.1080/00031305.1988.10475524

[8] Toplalova, I. \& Uzunova, M. (2017). Recognition of Similar Marble Textures through Different Neural Networks with De-correlated Input Data, International Journal on Advances in Intelligent Systems, ISSN:1942-2679, Volume10, Numbers3\&4, pp. 474-479.

[9] Widrow, B. \& Stearns, S. (2004). Adaptive Signal Processing, Prentice-Hall, Inc. Englewood Cliffs, N.J. 07632, pp.36-40.

[10] Gonzalez, R.C. \& Woods, R.E. (2008). Digital Image Processing 3rd Edition, Prentice Hall, India. 